\title{
Study the Effect of Immobilization on Electrostatic Potential for Methyl Orange Dye
}

\author{
Haider Shanshool Mohammed \\ Chemistry Department, College of Science,Al Muthanna University. \\ *Corresponding Author: haider.shanshool@yahoo.com
}

Received 27/9/2018, Accepted:18/1/2018, Published 26/4/2018

DOI: 10.52113/2/05.01.2018/38-43

\begin{abstract}
In the current study, the effect of the immobilization on electrostatic potential, $\mathrm{pH}$ and the absorbance of aqueous solutions of methyl orange that was immobilized on a modified chitosan polymer by glass wool were studied. It was found that the values of these parameters of the supported methyl orange are increased compared to unsupported samples. Furthermore, the electrostatic potential of the supported methyl orange is investigated in the presence of an aqueous solutions of $\mathrm{NaCl}$, where showed the value of the electrostatic potential is raised With increasing ionic strength of the sodium chloride solution and a decrease in the value of $\mathrm{pKa}$ to the methyl orange dye after immobilization while the results are inversely before immobilization methyl orange on a modified chitosan polymer by glass wool.
\end{abstract}

(C2018 Al Muthanna University. All rights reserved

Keywords: immobilization, electrostatic potential, methyl orange dye , chitosan

\section{Introduction:}

The development of porous materials with large specific surface area is currently an area of extensive research, particularly with regard to potential applications in area such as adsorption[1], in packing columns for chromatography[2], catalysis[3], sensors technology, and gas storage[4]. The most important families of these materials are (glass wool, cellulose, ion exchanger, polymer, silica gel etc.)[5].These materials are the most used materials and may have different internal pore size, depending on the surfactant and synthesis conditions[6, 7]. These nanofibers can form nonwoven textile mats, oriented fibrous bundles and even three-dimensional structured scaffolds, all with large surface areas and high porosity[8]. These nanofibers are of considerable interest for various kinds of applications.

Also in recent years, conducting polymers have received great attention in a wide range of areas due to their ease of generation, modification, environmental stability, excellent electrochemical, optical and magnetic properties, and thermo chemical characteristics. They are being widely used in wastewater treatment, electrochromic devices, catalysis, solar cells, nanoelectronics, metallic wires and biomedical devices[9-14]. Among the conducting polymers, Chitosan, a (1-4)- 
linked-2-amino-2-deoxy-D-glucopyranose

(Figure 1), is derived from chitin, one of the most abundant natural polysaccharides. Chitosan is well known for its nontoxic, biocompatible and biodegradable properties.

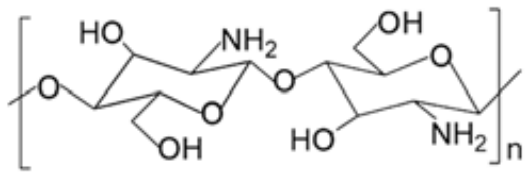

Fig.(1):The molecular structure of chitosan[15]

Therefore researchers interested in different spectral studies for acids and bases, such as spectra of UV[16, 17] , IR[18], NMR[19, 20] and spectra of mass[21] along with other studies included on the bloc[22, 23] and measure constants of ionization values[24, 25]. The researchers were sure that the voltammetry method[26].It is the best and used when studying the acidic pKa for many acids and bases and at different temperatures. It is curious to see in it Uncertainty because of pKa change with changes in temperature. The study that in our hands Interested in the effect of compensators, implied hydrogen bonds and factor of Obstruction stereoisomers on the $\mathrm{pKa}$ values for the acids and bases. And interested in as well in the effect of temperature and ionic strength on the electrolytes ionization constants. When Was acidic constants $\mathrm{Ka}$ is a form of equilibrium constants for reactions of ionized acids or bases [27]. Therefor in this study, the methyl orange dye was immobilized on the surface of the glass wool of the modified by the chitosan polymer. Where the methyl orange dye is a $\mathrm{pH}$ indicator frequently used in titrations because of its clear and distinct color change. Because it changes color at the $\mathrm{pH}$ of a mid- strength acid, it is usually used in titrations for acids. Unlike a universal indicator, methyl orange does not have a full spectrum of color change, but has a sharper end point. In a solution becoming less acidic, methyl orange moves from red to orange and finally to yellow with the reverse occurring for a solution increasing in acidity. The entire color change occurs in acidic conditions. In an acid $(\mathrm{pH}=3.1)$, it is reddish and in alkali $(\mathrm{pH}=4.4)$, it is yellow[28].

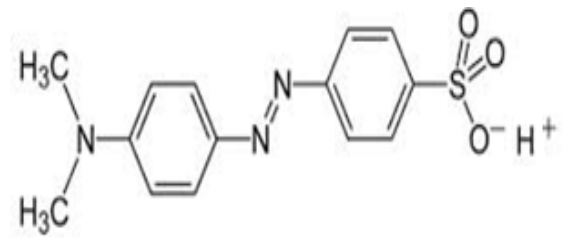

Fig.(2): Methyl orange dye

This dye can be immobilized on the surface of the glass wool modified by chitosan polymer through depending on the fiberglass characteristics. Quartz fiber refers to the silica content more than $99.90 \%, 1-15$ microns in size between the special fiber glass. Quartz fiber has the very high heat resistance, can at $1050{ }^{\circ} \mathrm{C}$ for long-term use, instant high temperature of $1700{ }^{\circ} \mathrm{C}$.Quartz fiber has excellent electrical insulation at the same time, its dielectric constant and dielectric loss factor is one of the best mineral fiber of all. Silica fibers are widely used in aerospace, military industry, semiconductor, oil chemical industry, building materials, electrical and electronic, heat insulation, high temperature filtration, etc. Our company production and operation of various specifications of quartz fiber yarn, quartz fiber cloth, quartz fiber, quartz fiber filament, casing quartz fiber, quartz fiber rope, quartz fiber quartz fiber products such 
as cotton. Products are widely used in aerospace, aviation, electronics, metallurgy, petrochemical, cables, electrical, atomic energy and other departments, mainly used as ablation resistant material, structure, materials, electrical materials, heat

\section{Experimental methods:}

2.1. Preparation different concentrations (20.0, 40.0, 60.0) ppm of aqueous solutions of methyl orange with a purity of $97 \%$ from Merck Company.

2.2. The characterization of glass wool was chosen by depended on general properties as light weight, a solid white support, and was able to adsorption of some compounds. It was modified with a chitosan (1- 4)- linked2-amino-2-deoxyD-glucopyranose Through immersion with equal weights pieces ( $1 \mathrm{~g} /$ piece) of glass wool in equal volume $(20 \mathrm{ml})$ of the polymer concentration $((1.0 \% \mathrm{~W} / \mathrm{V}) 1 \mathrm{~g}$ of polymer in $100 \mathrm{ml}$ of dist. water) for 24 hours at lab. temperature.

2.3. Submerged wool pieces in the chitosan polymer were washed 10 times with distilled water to remove the polymer that is not associated with glass wool.

2.4. Concentrations (20.0, 40.0, 60.0) ppm of methyl orange dye were immobilized on the surface of the glass wool that modified by chitosan polymer by immersing equal weights of glass wools that prepared in Step 3 in equal volumes of the dye for each concentration for 24 hours and at laboratory temperature. insulation, through materials, etc, therefor developed a general and comprehensive plan to study the effecting factors on the physical constants for the acidic compounds under study [29].

2.5. Use a Spectrophotometer (TRSP-72) to measure the absorbance at $\lambda \max 522$ $\mathrm{nm}$ for concentrations $(20.0,40.0,60.0)$ $\mathrm{ppm}$ of the aqueous solution of the methyl orange by using water as a solvent at laboratory temperature. Also use a $\mathrm{pH}$ meter 720-Germany to measure the $\mathrm{pH}$ value of the same aqueous solution for the methyl orange dye before immobilization.

2.6. Absorption and $\mathrm{pH}$ were measured for concentrations $(20.0,40.0,60.0) \mathrm{ppm}$ for the methyl orange dye with glass wool non modified by chitosan polymer through immersion equal weights pieces ( $1 \mathrm{~g} /$ piece) of glass wool in equal volume $(20.0 \mathrm{ml})$ for each concentration of the aqueous solution of methyl orange dye.

2.7.Absorption and $\mathrm{pH}$ values were measured by batch method for concentrations $(20.0,40.0,60.0) \mathrm{ppm}$ for aqueous solution of methyl orange dye that contain the glass wool modified by chitosan polymer $(1.0 \% \mathrm{~W} / \mathrm{V})$ that prepared in step 4.

2.8. The $\mathrm{pKa}$ and the ionic strength effect were calculated at titration each concentration of sodium chloride (0.001, $0.010,0.100) \mathrm{M}$ of the solution Contains (20 $\mathrm{ml}$ of $20.0 \mathrm{ppm}$ from methyl orang + glass wool non modified by chitosan polymer $+5 \mathrm{ml}$ sodium chloride ) with 
$(0.1 \mathrm{HCl} \quad \mathrm{M})$ before immobilization methyl orange dye.

2.9. The $\mathrm{pKa}$ and the ionic strength effect were calculated at titration each concentration of sodium chloride $(0.001$, $0.010,0.100) \mathrm{M}$ of the solution Contains $(20 \mathrm{ml}$ of $20.0 \mathrm{ppm}$ from methyl orang + $5 \mathrm{ml}$ of chitosan polymer $(1.0 \% \mathrm{w} / \mathrm{v})$ modified by glass wool $+5 \mathrm{ml}$ sodium chloride ) with $\left(\begin{array}{lll}0.1 & \mathrm{HCl} & \mathrm{M}\end{array}\right)$ after immobilization methyl orange dye.

\section{Results and Discussion:}

In this research studied the ability to immobilization methyl orange dye on a solid substrate (chitosan polymer modified on glass wool) as shown in Figure3.

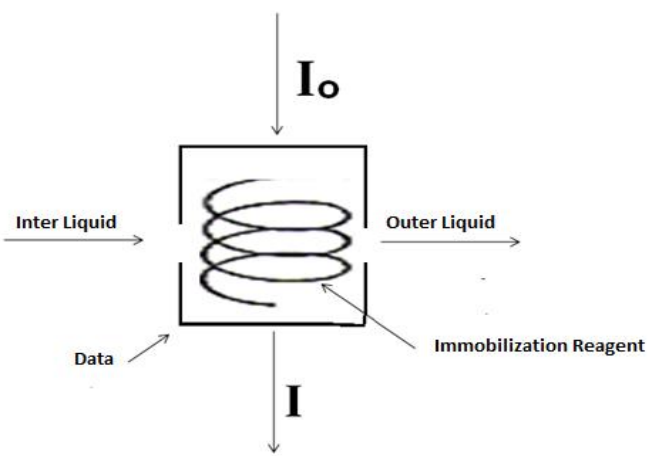

Fig.(3): Creating a sensors by depend on immobilized reagents indicator

Were also studied values of (Concentration $\mathrm{ppm}, \mathrm{pH}$, Absorption) for aqueous solutions of the methyl orange dye alone and with the unmodified glass wool and the results were compared with the modified glass wool by the chitosan polymer as shown in Table1, Figure $(4,5)[30]$.
Table 1: value of (Concentration ppm, $\mathrm{pH}$ ) for three states: Methyl orange in aqueous solution, Methyl orange with glass wool none modified and Methyl orange with glass wool modified by polymer Chitosan.

\begin{tabular}{|c|c|c|c|}
\hline State & $\begin{array}{c}\text { Concentratio } \\
\text { n ppm }\end{array}$ & pH & Absorption \\
\hline \multirow{3}{*}{1} & 20.0 & 6.87 & 0.441 \\
& 40.0 & 6.81 & 0.864 \\
& 60.0 & 6.72 & 0.912 \\
\hline \hline \multirow{3}{*}{3} & 20.0 & 6.91 & 0.452 \\
& 40.0 & 6.84 & 0.873 \\
& 60.0 & 6.77 & 0.925 \\
\hline \hline \multirow{3}{*}{3} & 20.0 & 3.62 & 0.125 \\
& 40.0 & 3.81 & 0.345 \\
& 60.0 & 3.95 & 0.531 \\
\hline
\end{tabular}

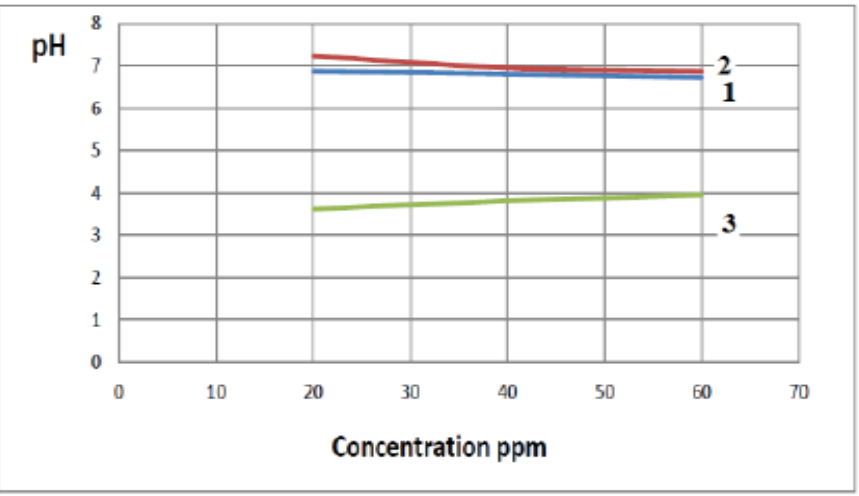

Fig.(4): The relation between $\mathrm{pH}$ and Concentrations ppm for three states: Methyl orange in aqueous solution, Methyl orange with glass wool none modified and Methyl orange with glass wool modified by polymer Chitosan.

In Figure $(4,5)$ was find the lines curve $(1$ and 2) at the top of the graph because the $\mathrm{pH}$ values and Absorption values of the aqueous solution of methyl orange as line curve (1) or aqueous solution of methyl orange with the 
glass wool non modified by chitosan polymer as line curve (2) was little difference in these values.

This indicates that the methyl orange dye did not immobilized on the glass wool non modified by chitosan polymer therefor chemical composition of the methyl orange dye in the first and second lines curve was not changed.

As for the third line curve (3), it is at the bottom of the graph where the $\mathrm{pH}$ and absorption values are the lowest as in Figure $(4,5)$. This indicates that the methyl orange dye immobilized on the modified chitosan polymer by glass wool.

The $\mathrm{pH}$ values are low due to the transfer of hydrogen ion from the methyl orange dye to the solution, which increases the acidity due to the formation of $\mathrm{H}_{3} \mathrm{O}^{+}$as in (Figure4). The absorbance values are low (Figure5) due to the decreases concentration of the methyl orange dye in the solution due to the immobilization methyl orange dye of chitosan polymer modified on the glass wool surface)[31].

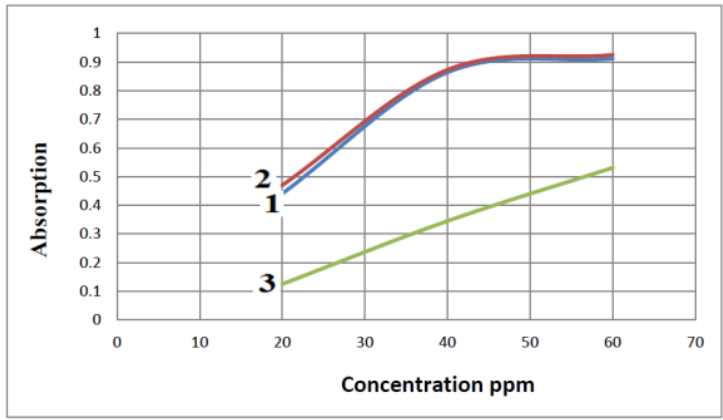

Fig.(5): The relation between Absorption and Concentrations ppm for three states: Methyl orange in aqueous solution, Methyl orange with glass wool none modified and Methyl orange with glass wool modified by polymer Chitosan.

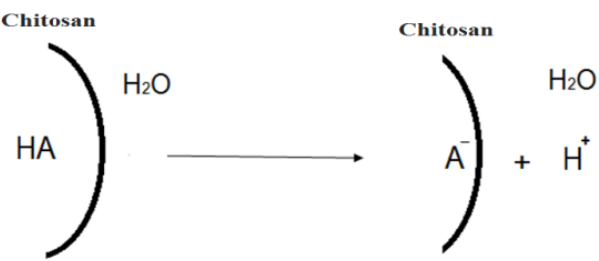

Fig.(6):. Electrostatic dissociation for immobilized indicator[32].

The electrostatic potential of the dye immobilized on the surface glass wool modified by the chitosan polymer was calculated by depend on ionic strength according to the following mathematical relation.

$$
\mathrm{I}=1 / 2 \sum \mathrm{Z}^{\mathrm{i} 2} \mathrm{C}^{\mathrm{i}}
$$

(I= ionic strength, $\mathbf{C}=$ concentration of $\mathrm{NaCl}$ ions, $\mathbf{Z}=$ charge of ions)

Through a set of physical constants (Gipps energy[33]) was found to calculate the electrostatic potential values of the immobilized methyl orange by depend on the values of $\mathrm{pKa}$

$$
\begin{gathered}
\left(p K_{a}\right)_{\text {эксп }}=p H=p K_{a}+\lg \frac{f\left(A^{-}\right)}{f(H A)} . \\
{[H A] \cong\left[A^{-}\right]}
\end{gathered}
$$

Moreover, we also have:

$\Delta \mathrm{Ga}=-\mathrm{RT} \ln \mathrm{Ka}=-2.3 \mathrm{RTpKa}$

Which relates the equilibrium constant with Gibbs free energy.

On the other hand the $\Delta \varphi=\Delta \mathrm{Ga} / \mathrm{n}$

( $\Delta \mathbf{G}=$ Gibbs free energy, $\boldsymbol{\varphi}=$ electrostatic potential, $\mathbf{R}=$ gas constant, $8.314 \mathrm{~J} \cdot \mathrm{mol}^{-1} \cdot \mathrm{K}^{-1}$ , $\mathbf{T}=$ absolute temperature, $\mathbf{K}_{\mathbf{a}}=$ equilibrium 
constant (unit less), $\mathbf{n}=$ number

of moles of electrons transferred in the reaction, $\mathbf{F}=$ Faraday constant $=96485$

$\mathrm{C} /$ mole (charge per mole of electrons).

There for $\Delta \varphi=-2.3 \mathrm{RTpKa} / \mathrm{n} \mathrm{F}$

$\mathrm{pKa}=-2.3 \mathrm{RT} \Delta \varphi / \mathrm{n} \mathrm{F}$

$\Delta \varphi=\varphi_{\mathrm{a}}-\varphi_{\mathrm{b}}$

$\varphi_{\mathrm{a}}=\varphi_{\mathrm{b}}+\Delta \varphi$

$\varphi_{\mathrm{b} \text { M.v }}=\mathrm{Z}$ e $/ 4 \pi \varepsilon \varepsilon_{0} \mathrm{r}=29.6 \mathrm{Mv}$

$\Phi_{\mathrm{a} M . \mathrm{v}}=29.6+\Delta \varphi$

(where is $\varphi_{\mathrm{a}}=$ electrostatic potential of methyl orange dye immobilized on the surface glass wool modified by the chitosan polymer, $\mathbf{Z}=$ Charge of ion $=+1$, $\mathbf{e}=$ Charge of electron $=1.6 * 10^{-19} \mathrm{col}, \boldsymbol{\varepsilon}=81$ where $\boldsymbol{\varepsilon}$ is the dielectric constant $, \quad \varepsilon_{0}=8,854 \cdot 10^{-12}$ where $\varepsilon_{0}$ is the dielectric constant of vacuum)[34].

Based on the mathematical equations above, the electrostatic potential and $\mathrm{pKa}$ was calculated by depended the ionic strength for the aqueous solution of the methyl orange dye alone and with the unmodified glass wool. The results were compared with the modified glass fiber by Chitosan polymer as shown in Table2, Figure7 and Figure8.
Table2: values of (pka, $\Delta \mathrm{pka}, \Delta \phi, \phi, \mathrm{I}$ ) for $20.0 \mathrm{ppm}$ of methyl orange solution in three states: Methyl orange in aqueous solution, Methyl orange with glass wool none modified

\begin{tabular}{|c|c|c|c|c|c|}
\hline & pka & $\Delta p k a$ & $\Delta \phi$ & $\phi$ & I \\
\hline \multirow[t]{4}{*}{1} & 6.87 & 0.00 & 0.000 & -59.200 & 0.000 \\
\hline & 6.91 & 0.04 & -2.348 & -61.548 & 0.001 \\
\hline & 7.11 & 0.24 & -14.088 & -73.288 & 0.010 \\
\hline & 7.23 & 0.36 & -21.132 & -80.332 & 0.100 \\
\hline \multirow{6}{*}{2} & & & & & \\
\hline & рка & $\Delta$ рка & $\Delta \phi$ & $\phi$ & 1 \\
\hline & 6.91 & 0.04 & -2.348 & -61.548 & 0.000 \\
\hline & 7.13 & 0.26 & -15.262 & -74.462 & 0.001 \\
\hline & 7.25 & 0.38 & -22.306 & -81.506 & 0.010 \\
\hline & 7.34 & 0.47 & -27.589 & -86.789 & 0.100 \\
\hline \multirow[t]{5}{*}{3} & pka & $\Delta$ pka & $\Delta \phi$ & $\phi$ & I \\
\hline & 3.62 & -3.25 & 190.775 & 131.575 & 0.000 \\
\hline & 3.53 & -3.34 & 196.058 & 136.858 & 0.001 \\
\hline & 3.41 & -3.46 & 203.102 & 143.902 & 0.010 \\
\hline & 3.33 & -3.54 & 207.798 & 148.598 & 0.100 \\
\hline
\end{tabular}

and Methyl orange with glass wool modified by polymer

Chitosan.

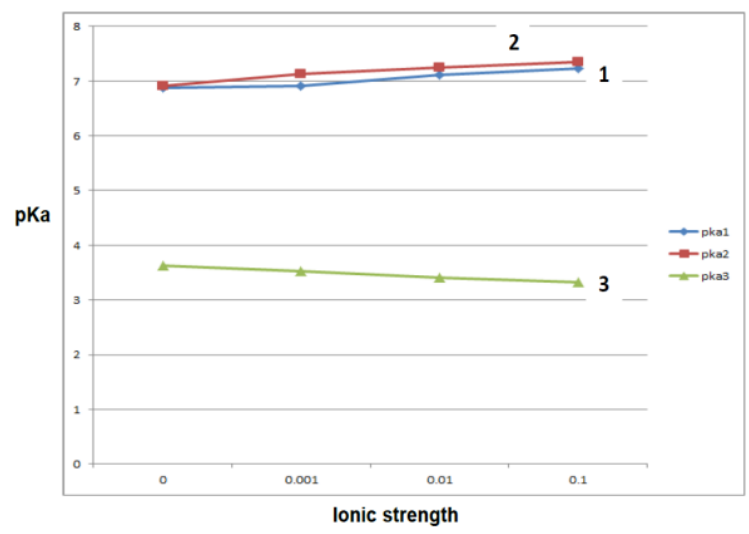

Fig.(7): The relation between $\mathrm{pKa}$ and ionic of strength of sodium chloride solution at titration20.0 ppm of methyl orang with ( 0.1 $\mathrm{HCl} \mathrm{M}$ ) for three states: Methyl orange in aqueous solution, Methyl orange with glass wool none modified and Methyl orange with glass wool modified by polymer Chitosan. 
As for the third line curve (3), it is at the bottom of the graph where the pKa values are the lowest as in (Figure 7). This indicates that the methyl orange dye immobilized on the modified chitosan polymer by glass wool.

The $\mathrm{pKa}$ values are low due to the transfer of hydrogen ion from the methyl orange dye to the solution, which increases dissociation constant due to the formation of $\mathrm{H}_{3} \mathrm{O}^{+}$ion due to the immobilization methyl orange dye of chitosan polymer modified on the glass wool surface.

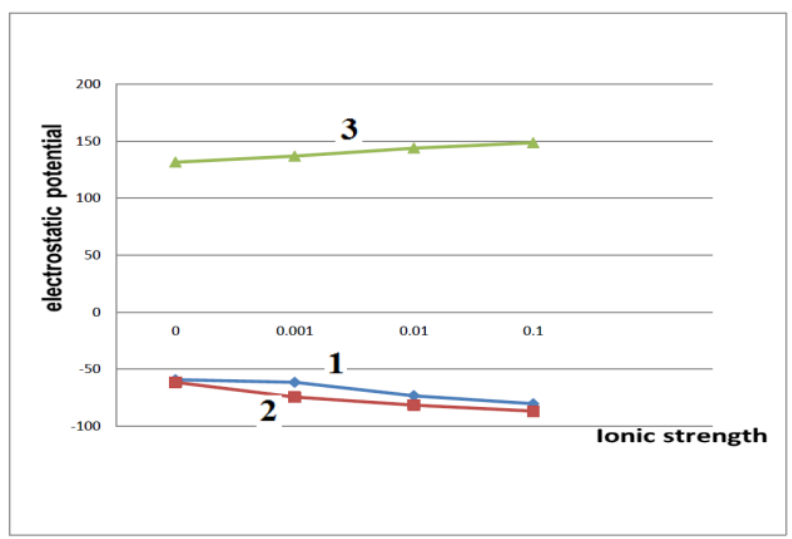

Fig.(8): The relation between electrostatic potential and ionic strength of sodium chloride solution at titration $20.0 \mathrm{ppm}$ of methyl orang with $(0.1 \mathrm{HCl} \mathrm{M})$. for three states: Methyl orange in aqueous solution, Methyl orange with glass wool none modified and Methyl orange with glass wool modified by polymer Chitosan.

During the study the effect of electrostatic potential of the methyl orange dye based on the ionic strength of the solution it was observed that the line of curve (3) as in (Figure 8) is at the top of the graph indicating that the methyl orange dye immobilized on the modified chitosan polymer by glass wool as a result of increasing the value of the electrostatic voltage by increasing the ionic strength of the solution causing the ionic transfer and obtain the chemical adsorption between the methyl orange dye and chitosan polymer modified by glass wool .In the other hand we find the lines curve (1 and 2) at the bottom of the graph because don't obtain the chemical adsorption between the methyl orange dye and glass wool non modified by chitosan polymer because the electrostatic potential of the methyl orange dye as line curve (1) and aqueous solution of methyl orange with the glass wool non modified by chitosan polymer as line curve (2) was little difference This indicates that the methyl orange has not immobilized on the glass wool non modified by chitosan polymer because the chemical adsorption did not occur therefor ionic transition did not occur[35].

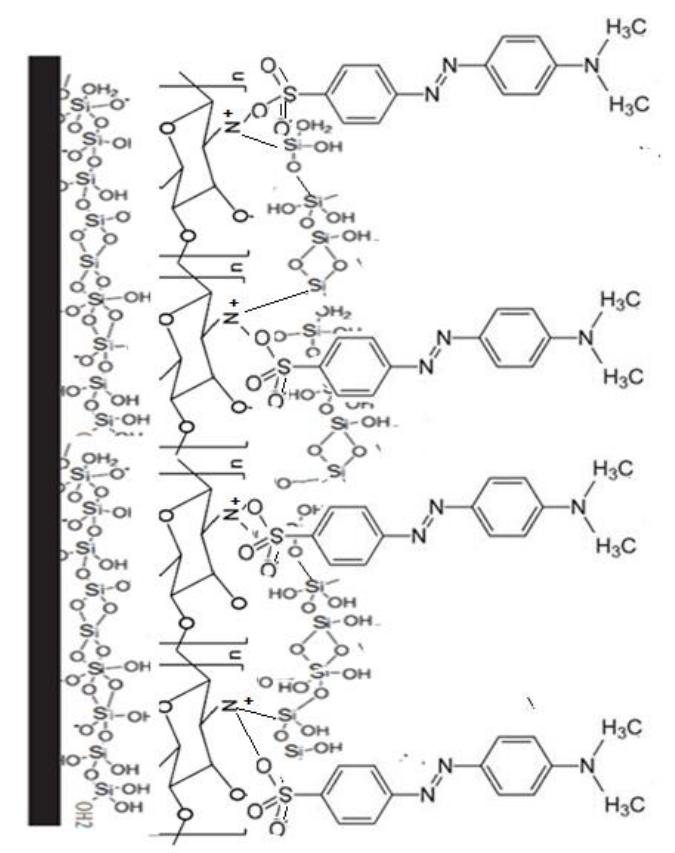

Fig.(9):. Suggested form of Glass wool with polymer Chitosan + methyl orang indicator [36,37,38]. 


\section{Conclusions:}

When immobilized of methyl orang indicator, the $\mathrm{pH}$ changes to the a Acidic medium and the value of $\Delta \mathrm{pH}$ transition connected with the effect of micro phase of the electrostatic potential for methyl orang indicator while the value of $\Delta \mathrm{pKa}$ of methyl orang indicator increasing with effect of ionic strength before immobilization and decreasing with increasing of ionic strength after immobilization with quartz modified by polymer Chitosan also the effect of the electrostatic potential for methyl orang indicator decreasing before immobilization and increasing after immobilization with quartz modified by polymer Chitosan

\section{References}

[1] Branton PJ, Hall PG, Sing KSW. J Chem Soc Chem Commun (1993); 16:1257-8.

[2] A. Kurganov, K. Unger, T. Issaeva, J. Chromatogr. A 753 (1996) 177.

[3] On DT, Desplantier-Giscard D, Danumah C, Kaliaguine S. Appl Catal A (2001);22: 299-357.

[4] Meynen V, Cool P, Vansant EF. Micropor Mesopor Mater (2009); 125: 170-223.

[5] B. Naik, N.N. Ghosh, Recent Pat. Nanotech. 3 (2009) 213.

[6] T. Benamor, L. Vidal, B. Lebeau, C. Marichal, Micropor. Mesopor. (2012) Mater. $153,100$.

[7] Y.Y. Fahn, Langmuir 21 (2005) 431.
[8] Hunley M. T., Long T. E.: Electrospinning functional nanoscale [13] Z. Chen, Y. Takei, B. A. Deoye and T. Nagaoka, Analyst, (2000), 125, 2249-2254.

[14] M. Zhao, X. Wu and C. Cai, J. Phys. Chem. C, (2009), 113, 49874996.

[15] Mi F-L., Tan Y-C., Liang H-F., Sung H-S.: In vivo biocompatibility and degradability of a novel injectable- chitosan-based implant. Biomaterials, (2002), 23, 181-191 . [16] A.S.P. Azzouz and M.M.H. AlNiemi, Z. Phys. Chem., 2005, 219, 1591.

[17] A.S.P. Azzouz and N.A. AlAzzawi, J. Edu. Sci., (2004), 16, 93. 
[18]A.S.P.Azzouz and Kh.AL- Niemi, J.Edu.Sci,2004,16,59.

[19] Albert and E.P. Serjeant, The Determination of,, Ionization Consta nts,2nded.,Chapman and Hall, London,(1971).

[20] A.S.P. Azzouz, N.A. Al- Azzawi, Iraqi J. Chem., (2005), (Abstract).

[21] Ali Latif Hamid,industrial pollution, Mosul University, (1987) [22] J.cenens, Schoonhydt RA(1988). [23] Edward G. Fundamentals of high performs concrate, Nawy, John sons and wily page 310 ISBN 978-0-47138555-4.

[24]L.N. Ferguson and I. Kelly, J.Amer. Chem. Soc., (1951), 73, 3707. [25] A.A. Saeed, A.W.A. Sultan, S.A. Selman and N.A. Abood, Can. J. Spectrosc., (1983), 28, 104.

[26] A.S.P. Azzouz, Z. Phys. Chem., (2002), 216, 1 ed., Chapman and Hall, London.

[27] A.S.P. Azzouz, N.A. Al- Azzawi, Iraqi J. Chem., (2005), (Abstract). [28] J.cenens, Schoonhydt RA (1988). [29]Nawy, Edward G. (2001). Fundamentals of highperformance concrete (2 ed.). John Wiley and Sons. p. 310. ISBN 978-0471-38555-4.

[30] A.S.P. Azzouz, Spectroscopy Letters, (1995), 28, 1.

[31] R.F. Cookson,Chemical Reviews, 1974, 74, 1.

[32]Chan,T.-H.; Melnyk, A. Kinetics and mechanism of the sulfoxidesilane reaction. J. Am. Chem. Soc. (1970), 92, 3718-3722.
[33]J.W. Gibbs, "A Method of Geometrical Representation of the Thermodynamic Properties of Substances by Means of Surfaces," Transactions of the Connecticut Academy of Arts and Sciences 2, Dec. 1873, pp. 382-404 (quotation on p. 400).

[34]Costa, F.; Amabile, C.; Monorchio, A.; Prati, E. (2011). "Waveguide Dielectric Permittivity Measurement Technique Based on Resonant FSS Filters". IEEE Microwave and Wireless Components Letters. 21 (5):273. doi:10.1109/LMW C.2011.2122303.

[35]Salih,Jalal ,Mohammed, Electrical chemistry, Bagdad university, (1992).

[36] Wang, F. et al. A highly efficient photocatalytic system for hydrogen production by a robust hydrogenase mimic in an aqueous solution. Angew (2011). Chem. Int. Ed. 50, 3193-3197.

[37] Goerl,U.; Hunsche, A.; Mueller, A.; Koban, H.G. Investigations into the silica/silane reaction system .Rubber Chem Technol.(1997), 70, 608-623.

[38] James, E.M. Overview of Siloxane Polymers. In Silicones and Silicone-Modified Materials;

American Chemical Society: Washington, DC, USA, (2000); 729,1-10. 